\title{
Divertículo duodenal perforado. Reporte de caso
}

\author{
Perforated duodenal diverticulum. Case Report \\ Gustavo Armand Ugon $^{1}$,Daniel González ${ }^{2}$,Pilar Arciénega ${ }^{3}$, Gabriela Rodríguez ${ }^{4}$
}

DOI: $10.31837 /$ cir.urug/3.2.3

Recibido: 18 de diciembre de 2018

Aceptado:

05 de abril de 2019

\section{Resumen}

La perforación de un divertículo duodenal (Dd) es una complicación excepcional, no presenta clínica patognomónica.

Paciente de 60 años, mujer, sin antecedentes de dispepsia ulcerosa. Dolor epigástrico intenso, transfixiante, 8 horas de evolución. Dolor a la palpación de hipocondrio derecho.

Paraclínica humoral: leucocitosis 14.200, resto normal. Tomografía: Dd perforado y retroperitonitis.

Mediana supraumbilical. Decolamiento duodenocéfalo-pancreático, identificación y resección del Dd, rafia duodenal en dos planos (poliglactina-910, surjet), toilette y drenaje retroperitoneal.

Postoperatorio: evolución sin complicaciones, alta a la semana.

Resolvimos la perforación mediante duodenorrafia, no realizamos exclusión duodenal ni gastro-yeyunoanastomosis (estos dos últimos están incluidos en el tratamiento más recomendado).

Palabras clave: Diverticulitis, divertículo duodenal, perforación

\section{Abstract}

The perforation of a duodenal diverticulum (Dd) is an exceptional complication, it does not present a pathognomonic clinic.

60 -years-old woman, with no history of ulcerative dyspepsia. Intense epigastric pain, transfixing, 8 hours of evolution. Pain on palpation of right hypochondrium.

Humoral paraclinical: leukocytosis 14,200, normal rest. Tomography: perforated Dd and retroperitonitis.

Supraumbilical medium. Decodeduodenumcephalo-pancreatic, identification and resection of theDd, duodenal raffia in two planes (polyglactin910, surjet), toilette and retroperitoneal drainage.

Postoperative: evolution without complications, discharge a week.

We solved the perforation by duodenorraphy, we did not perform duodenal exclusion orgastrojejunum-anastomosis (the latter two are included in the most recommended treatment).

Keywords: diverticulitis, duodenal diverticulum, perforation

\footnotetext{
${ }^{1234}$ Clínica Quirúrgica 3. Hospital Maciel, Facultad de Medicina, Universidad de la República. Montevideo, Uruguay gusad@adinet.com.uy
} 


\section{Introducción y marco teórico}

Los divertículos duodenales (Dd) aparecen como "curiosidad anatómica” en las descripciones de Chomen (1710) y Morgagni (1761). Posteriormente como hallazgo quirúrgico e imagenológico. ${ }^{1}$

En 1907 Bassett describió la primera perforación duodenal evidenciada al realizar una autopsia. ${ }^{1.2}$

El duodeno es, después del colon, el segundo sitio de asiento de los divertículos intestinales.

Los Dd pueden ser congénitos o adquiridos. Los congénitos son consecuencia de invaginaciones de la pared duodenal durante el desarrollo embrionario, están formados por todas las capas del órgano. Los adquiridos, sea por pulsión o tracción, son los más frecuentes, están formados por mucosa y submucosa herniadas a través de la capa muscular. ${ }^{3.4}$

De forma redondeada, y con un diámetro que varía entre 0,5 y $5 \mathrm{~cm}$, son generalmente únicos.

Presentan igual incidencia en ambos sexos. Su prevalencia es del 22\% de la población y aumenta con la edad, son excepcionales antes de los 30 años. ${ }^{2.3 .4 .5}$

El 95\% de los Dd están localizados en la concavidad del asa duodenal, pudiendo ser retro, intra o pre-pancreáticos, siendo estos últimos excepcionales, por ello la perforación de un Dd origina una retro-peritonitis que rápidamente puede evolucionar a la sepsis. ${ }^{1.4 .6}$

El 75\% de los Dd se topografía en la segunda porción y en la proximidad de la papila (sitio donde la pared duodenal es más débil, consecuencia de la presencia de la “ventana duodenal”). Siguen en frecuencia: tercera porción (15\%), cuarta (9\%), primera y ángulo de Treitz (1\%). ${ }^{4.6}$

Generalmente asintomáticos y sin clínica patognomónica, el 5-10\% pueden complicarse con; hemorragia, ictericia obstructiva, perforación, oclusión (orden decreciente de frecuencia). ${ }^{1.3 .4 .5 .6}$

Ninguna intervención quirúrgica es necesaria ante un Dd no complicado. Menos del 1\% de los Dd requerirán cirugía. ${ }^{1.2 .3 .6 .}$

La perforación de un Dd es una complicación poco frecuente y de difícil diagnóstico, ya que no tiene una sintomatología específica. Es grave, porque origina una retro-peritonitis. Tiene una mortalidad del 8\%. ${ }^{1.4}$

La tomografía abdominal con contraste endoluminal y endovascular permite el diagnóstico del divertículo y de la complicación. En la casi totalidad de los casos se solicita con planteo diagnóstico presuntivo de otras patologías del hemiabdomen superior. ${ }^{1.2 .4}$.

Se han publicado 162 casos hasta 2012, siendo las causas más frecuentes de perforación la diverticulitis (62 a 69\%) y la entero-litiasis (10\%). Otras causas son; iatrogénica (5\%), es rara la perforación por cuerpo extraño $(1,2 \%)^{1.4 .5 .7}$

El tratamiento quirúrgico más utilizado es la diverticulectomía simple más duodenorrafia y drenaje del espacio retroperitoneal a lo que se asocia una exclusión duodenal y gastro-yeyuno anastomosis. ${ }^{1.2 .4 .5}$ Otras alternativas son la diverticulectomía simple más duodenorrafia y drenaje retroperitoneal sin otro procedimiento quirúrgico asociado, o la diverticulostomía. ${ }^{1.4 .5}$

Se han reportado casos de abordaje laparoscópico y de tratamiento conservador exitoso en base a drenaje percutáneo, antibioticoterapia y reposo digestivo. ${ }^{1.5 .8}$ 
Las complicaciones postoperatorias más frecuentes son la fístula duodenal o pancreática, la estenosis de la ampolla de Vater, la estenosis o la lesión de la vía biliar principal (debe tenerse especial cuidado que no quede comprometida por la rafia duodenal), la pancreatitis. ${ }^{3.4 .7}$

\section{Caso clínico}

Mujer, 60 años, sin antecedentes de dispepsia ulcerosa. Consulta por dolor abdominal de inicio brusco sin relación con las ingestas, tipo puntada, intenso, en epigastrio, transfixiante. 8 horas de evolución. Acompañado de nauseas, no vómitos. Eupneica, bien perfundida, apirética. Dolor intenso a la palpación de hipocondrio derecho, sin defensa ni ocupación, que requirió analgésicos mayores endovenosos.

Paraclínica humoral: Leucocitosis 14.200, resto normal.

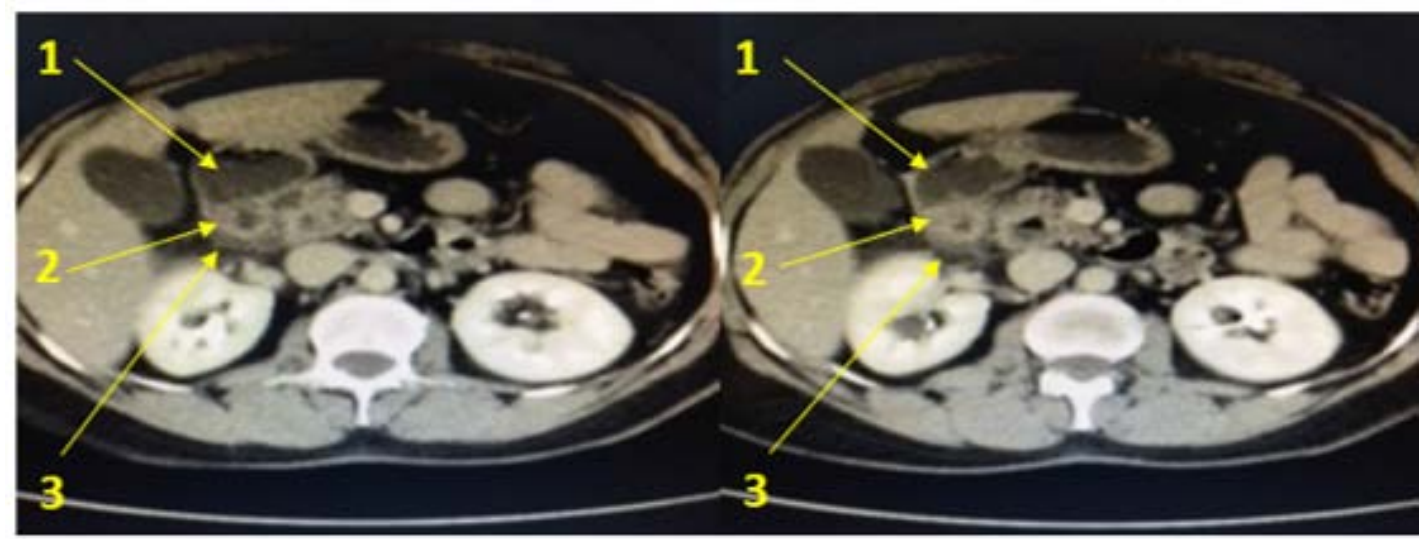

Figura 1:

1) Duodeno II, 2) Diverticulo duodenal, 3) Aire retroperitoneal

Tomografía (fig.1): alteración inflamatoria de la grasa periduodenal y pequeñas burbujas de gas retroperitoneal adyacentes a imagen de 27x23mm inmediatamente por detrás de la segunda porción duodenal (D2), bien delimitada, contiene líquido, contraste y múltiples burbujas. Planteo diagnóstico: Dd perforado.

Abordaje laparotómico: mediana supraumbilical. No hay líquido libre ni aire en cavidad peritoneal, se evidencia inflamación de D2 y periduodenal.

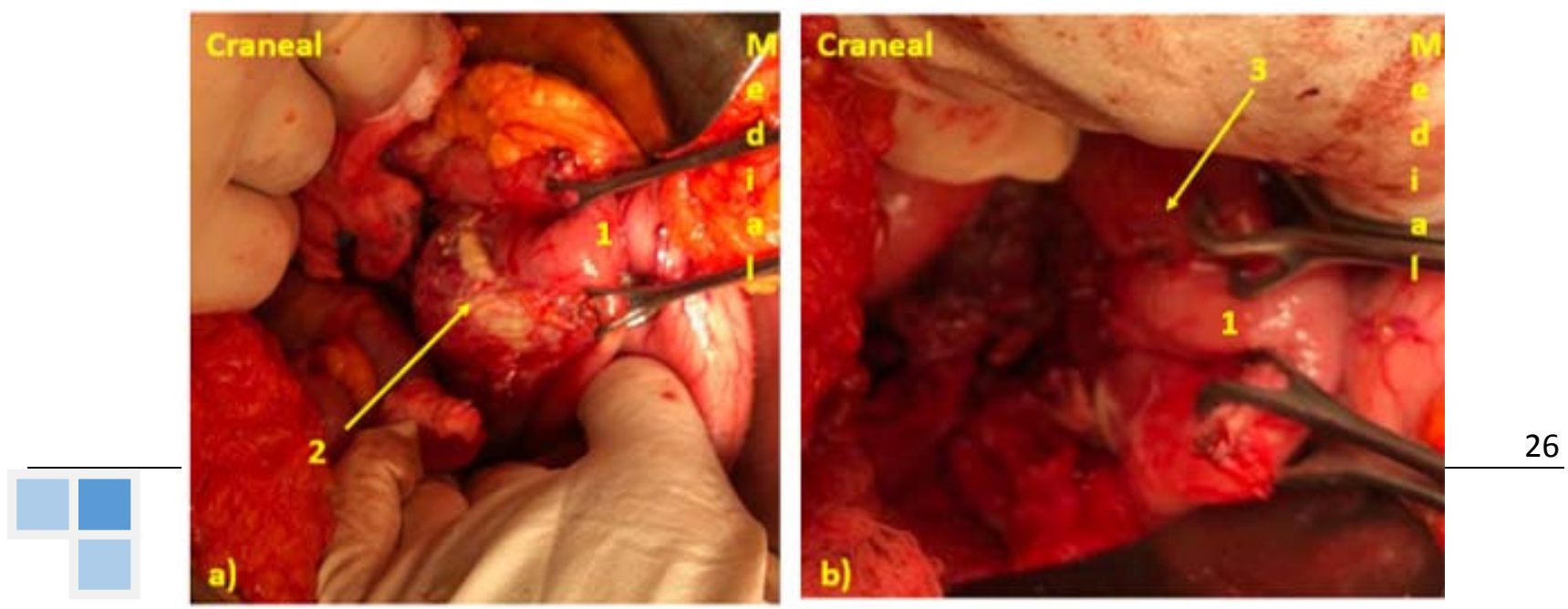

Figura 2:

a) decolamiento duodeno céfalo pancreático. Tejido peri pancreático inflamado.

b) decolamiento duodeno céfalo pancreático. Tejido peri pancreático retirado, se expone diverticulo duodenal. 1) duodeno II, 2) tejido inflamado, 3) diverticulo duodenal, 
Decolamiento duodeno-céfalo-pancreático parcial, supramesocólico, confirma Dd redondeado, perforado en su ápex, con retro-peritonitis localizada (fig.2).

Divertículo topografiado en mitad superior de D2, cara posterior, cerca de la convexidad del asa. Se identificó el colédoco retro duodenopancreático.

Resección del divertículo duodenal (se envía a anatomía patológica diferida). Rafia duodenal en dos planos (surjet de poliglactina-910), lavado del área con suero fisiológico, colocación de drenaje de látex enfrentado a la rafia duodenal. Se dejó sonda naso gástrica (SNG).

Postoperatorio en apirexia. SNG sin retención, se retira a las 36 horas y se restablece progresivamente la vía oral. Antibioticoterapia: ampicilina-sulbactam 1,5 g endovenosos cada 6 horas (7 días). Analgésicos de potencia intermedia, endovenosos, reglados, (4 días). Drenaje con gasto casi nulo, serohemático, se retira a las 48 horas. Herida operatoria sin complicaciones. Alta a los 7 días.

Anatomía patológica informa: diverticulitis duodenal con área necrosada, sin malignidad.

\section{Comentario final}

En el caso clínico presentado, perforación diverticular con retro-peritonitis localizada de pocas horas de evolución, el dolor intenso desde el inicio motivó la indicación de tomografía, con la que realizamos diagnóstico. Las pocas horas entre la perforación y su resolución quirúrgica favorecieron la buena evolución postoperatoria ya que la retro-peritonitis estaba limitada al área del asa duodenal.

A diferencia de lo recomendado, resolvimos la perforación diverticular mediante diverticulectomía, duodenorrafia y drenaje, sin realizar exclusión duodenal y gastro-yeyuno anastomosis.

La topografía del Dd encontrado, próximo a la convexidad del asa duodenal, es excepcional.

\section{Bibliografía}

1- De Miguel, M; Otegui, I; Cuco, S; Salvador, P; Riaño, M; Fidalgo, M. Diverticulitis duodenal perforada. Rev Port Cir 2014;(31):41-7.

2- Meyer, C; Rohr, S: Firtion, O. Chirugie des diverticulesduodenaux. EncyclMedChir. Elsevier, Paris-France, 1996.Techniques chirurgicales - Appareildigestif, 40-410

3- Mathis, K; Farley, D. Operativemanagement of symptomatic duodenal diverticula. Am J Surg. 2007:93:305-8.

4- De Miguel, M; Otegui, I; Vesga, A; Marín, G; Acevedo, A; Esquíroz, I, et al. Diverticulitis duodenal perforada. Cir. Esp. 2014;92(EspecCongr):983. 
5- Mayo, M; Pacheco, J; Roldan, S; Fernández, J. Diverticulo duodenal simulando neoplasia quística del páncreas. RevChilCir 2018;70(2):164-7.

6- Egawa, N; Anjiki, H; Takuma, K; Kamisawa, T. Juxtapapillary duodenal diverticula and pancreatobiliarydisease. DigSurg. 2010;27:105-9.

7- Thorson, C; Paz, P; Roeder, R; Sleeman, D; Casillas, V. Theperforated duodenal diverticulum. ArchSurg 2012;147:81-8.

8- Favre, J; López-Tomassetti, E; Ceballos, J; Santana, L; Hernández, J. Divertículo duodenal perforado por cuerpo extraño. RevEspEnfermDig. 2013;105/6:368-9. 